\section{Prostate cancer: adenovirus-mediated imaging of sentinel node metastasis}

Lymph node status in prostate cancer is an important predictor of outcome, but currently, no reliable, noninvasive methods exist to detect lymph node metastasis. Now, researchers at the University of California, Los Angeles, describe the use of an adenovirus vector to allow direct imaging of metastasis in sentinel lymph nodes. Their study used a mouse xenograft model of human prostate cancer.

Burton et al. developed adenovirus vectors that express optical and PET reporter genes under the control of an amplified PSA promoter to permit prostate-specific imaging. Adenoviruses preferentially infiltrate the lymphatic system, so injection of these vectors into the forepaws of mice bearing human prostate tumors derived from the aggressive LAPC-9 cell line allowed specific bioluminescent and PET imaging of metastatic lymph nodes. PET detected nodes $2.5 \mathrm{~mm}$ in diameter. Peritumoral virus injection, in a manner analogous to the lymphoscintigraphy technique, permitted visualization of PET signals both from the tumor and from metastases within the draining axillary lymph node.

The authors conclude that lymphangiography using the adenoviral gene transfer technique promises to facilitate accurate, noninvasive detection of lymph node metastasis. In addition, one of the reporter genes carried by the vector, the herpes simplex thymidine kinase gene, has also previously been shown to sensitize tumor cells to ganciclovir, which opens up the possibility of using these adenoviral vectors for both diagnostic imaging and suicide gene therapy.

Original article Burton JB et al. (2008) Adenovirusmediated gene expression imaging to directly detect sentinel lymph node metastasis of prostate cancer. Nat Med 14: 882-888

\section{Infertility due to C. trachomatis and Mycoplasma is a result of sperm DNA fragmentation}

Chlamydia trachomatis is a major cause of infertility, but the exact mechanism of its effect on sperm has been unclear. Now, a Spanish study has found that the sperm of men infected with C. trachomatis have increased levels of DNA fragmentation.

Gallegos et al. compared sperm from 143 infertile patients infected with $C$. trachomatis (all of whom were also positive for Mycoplasma infection) with sperm from 50 fertile control individuals. Although sperm motility, concentration and morphology were significantly worse in patients than in controls, these characteristics were within the WHO-defined normal range for most patients $-73 \%$ were normozoospermic by WHO criteria. However, analysis of sperm DNA showed that a mean of $35 \%$ of sperm cells had fragmented DNA in the patient group, compared with $11 \%$ in the control group. After a mean of 3.8 months of antibiotic and anti-inflammatory treatment in a subgroup of 95 patients, the mean frequency of sperm with DNA fragmentation decreased from $38 \%$ to $24 \%$. Of other sperm characteristics, only morphology showed an improvement.

Of 16 couples who attempted conception during antibiotic treatment, $12.5 \%$ were successful; however, of 14 couples who attempted pregnancy 3-6 months after therapy, $84.7 \%$ were successful. The mean percentage of sperm with DNA fragmentation was significantly lower in the second group (32\% vs $43 \%$ of cells).

The authors conclude that $C$. trachomatis and Mycoplasma infection adversely affects fertility by increasing sperm DNA fragmentation. Antibiotic therapy reduces this damage and can restore fertility in the individuals affected.

Original article Gallegos G et al. (2008) Sperm DNA fragmentation in infertile men with genitourinary infection by Chlamydia trachomatis and Mycoplasma. Fertil Steril 90: 328-334

\section{A poxvirus vaccine improves survival in men with castration- resistant prostate cancer}

In 2006, a prospective study of 42 men with nonmetastatic, castration-resistant prostate cancer reported that immunization with a poxvirus vaccine improved time to treatment failure. Madan et al. evaluated these same patients 6.5 years after the initiation of therapy, and have shown that vaccination also prolongs overall survival in this setting. 